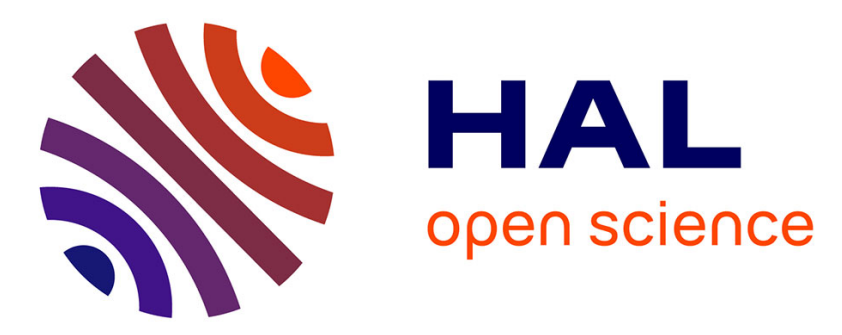

\title{
Infinite Dimensional Port Hamiltonian Representation of Chemical Reactors
}

Weijun Zhou, Boussag Hamroun, Yann Le Gorrec, Françoise Couenne

\section{To cite this version:}

Weijun Zhou, Boussag Hamroun, Yann Le Gorrec, Françoise Couenne. Infinite Dimensional Port Hamiltonian Representation of Chemical Reactors. 4th IFAC Workshop on Lagrangian and Hamiltonian Methods for Non Linear Control, LHMNLC'12., Aug 2012, Bertinoro, Italy. pp.1-6. hal00803504

\section{HAL Id: hal-00803504 https://hal.science/hal-00803504}

Submitted on 22 Mar 2013

HAL is a multi-disciplinary open access archive for the deposit and dissemination of scientific research documents, whether they are published or not. The documents may come from teaching and research institutions in France or abroad, or from public or private research centers.
L'archive ouverte pluridisciplinaire $\mathbf{H A L}$, est destinée au dépôt et à la diffusion de documents scientifiques de niveau recherche, publiés ou non, émanant des établissements d'enseignement et de recherche français ou étrangers, des laboratoires publics ou privés. 


\title{
Infinite Dimensional Port Hamiltonian Representation of Chemical Reactors
}

\author{
W. Zhou* B. Hamroun* Y. Le Gorrec** F. Couenne* \\ * LAGEP, LAGEP, UMR CNRS 5007, University of Lyon1, \\ Villeurbanne, France, (e-mails: \\ \{zhou;hamroun;couenne\}@lagep.univ-lyon1.fr). \\ ** AS2M ENSMM Besançon, Besançon, France (e-mail: \\ yann.le.gorrec@ens2m.fr)
}

\begin{abstract}
Infinite dimensional Port Hamiltonian representation of non isothermal chemical reactors is proposed in the case of mass transport diffusion and chemical reaction without convection. The proposed approach uses thermodynamic variables. The presentation is given for one dimensional spatial domain by using the internal energy and the opposite of the entropy as hamiltonian functions.
\end{abstract}

Keywords: Port Hamiltonian Systems, Distributed Systems, Irreversible Thermodynamics

\section{INTRODUCTION}

Models of chemical reactors vary in details from ordinary differential equations for the modeling of a Continuous Stirred Tank Reactor (CSTR) to a large set of coupled Partial Differential Equations (PDEs) for tubular reactors. Due to the strong coupling between transport and reaction phenomena and the nonlinear dependency of the reaction rates on the temperature and the concentrations, these models exhibit very complex behavior. Usually, models of chemical reactors are based on the conservation of extensive quantities, namely mass, energy and momentum and balance of each species.

In this paper we propose a Port Hamiltonian representation of a distributed reactor for control purpose. Port Hamiltonian representation of CSTRs has already been proposed in Hoang et al. (2011) and has been used for control using passivity based methods. The authors proposed two representations: the first one is based the total energy (i.e. the internal energy) and the second one uses the opposite of entropy as Hamiltonian function.

In this paper we consider the distributed parameter case. We discuss how the state variables have to be chosen such that geometric properties of the model are emphasized. Indeed, in the Port Based Modeling approach the number of variables is increased in view of pointing out the fundamental geometric power conserving interconnection structure of the distributed parameter system. This power conserving interconnection structure is also called Stokes Dirac structure (see Maschke and Van der Schaft (2005)). We show that this approach is relevant for the modeling of systems such the tubular reactor. Precisely, in this paper, the geometric structure is used for the modeling of a process where some diffusion phenomenon and chemical reactions take place.

In section 2 we briefly recall the general port hamiltonian formulation of distributed parameter system. In section 3 , using the irreversible thermodynamics, we propose some natural variable pairings that can be used in the case of distributed parameters reactors. In section 4 we present the port hamiltonian formulation of the reactor.

\section{INFINITE DIMENSIONAL PORT HAMILTONIAN SYSTEMS}

The port Hamiltonian formulation of distributed parameter systems is based on a generalization of the finite dimensional Dirac structure Courant (1990); Dorfman (1993). This geometrical structure links the flow variables and the effort variables through a skew symmetric operator which interprets the power conservation principle. First, let us briefly recall the hamiltonian formulation of two energy domains in 1 -Dimensional case $(\mathcal{Z}=[a, b])$. Next, we present a specific extension to dissipative systems.

Consider the flow variables $f=\left(f_{q}, f_{p}\right)$ given as time derivative of the extensive variables $x=(q, p) \in \mathbb{R}^{2}$ $\left(f_{q}=-\frac{\partial q}{\partial t}, f_{p}=-\frac{\partial p}{\partial t}\right)$ and the effort variables $e=\left(e_{q}, e_{p}\right)$ given as the variational derivatives of the Hamiltonian $H$ $\left(e_{q}=\delta_{q} H, e_{p}=\delta_{p} H\right)$. The canonical Dirac structure is then given by (see Maschke and Van der Schaft (2005); Le Gorrec et al. (2005)):

$$
\begin{gathered}
\left(\begin{array}{c}
f_{q} \\
f_{p}
\end{array}\right)=\mathcal{J}\left(\begin{array}{c}
e_{q} \\
e_{p}
\end{array}\right)+\mathcal{B}\left(f_{\mathcal{Z}}^{c}\right) \\
e_{\mathcal{Z}}^{c}=-\mathcal{B}^{\star}\left(\begin{array}{c}
e_{q} \\
e_{p}
\end{array}\right) \\
e_{\partial}^{a}(t)=-e_{q_{\mid z=a}} f_{\partial}^{a}(t)=e_{p_{\mid z=a}} \\
e_{\partial}^{b}(t)=-e_{q_{\mid z=b}} f_{\partial}^{b}(t)=e_{p_{\mid z=b}}
\end{gathered}
$$

with $\mathcal{J}=\left(P_{0}+P_{1} \partial_{z}\right), P_{1}=\left(\begin{array}{ll}0 & 1 \\ 1 & 0\end{array}\right)$ and $P_{0}=0_{2} . e_{\partial}$ and $f_{\partial}$ are the boundary variables given as a restriction of distributed effort variables on the boundary and $f_{\mathcal{Z}}^{c}$ is the external distributed flow control with its conjugate effort $e_{\mathcal{Z}}^{c} \cdot \mathcal{B}$ is a non differential linear operator with its 
adjoint $\mathcal{B}^{\star}$. The power product associated with this Dirac structure is given by

$$
\begin{aligned}
<\left[\begin{array}{c}
e \\
e_{\mathcal{Z}}^{c} \\
e_{\partial}
\end{array}\right] \mid\left[\begin{array}{c}
f \\
f_{\mathcal{Z}}^{c} \\
f_{\partial}
\end{array}\right]>: & =<e, f>_{L^{2}}+<e_{\mathcal{Z}}^{c}, f_{\mathcal{Z}}^{c}>_{L^{2}} \\
& +<e_{\partial}^{b}, f_{\partial}^{b}>_{R^{2}}-<e_{\partial}^{a}, f_{\partial}^{a}>_{R^{2}}
\end{aligned}
$$

where $<e, f>_{L^{2}}=\int_{a}^{b} e^{T} f d z$ is the duality product in $L^{2}[a, b]$ (power product) and $<e, f>_{R^{2}}=e^{T} f$ is the scalar product in $R^{2}$. This power product (4) expresses the conservation of energy of the system.

From a geometric point of view the Dirac structure is defined as a subspace $\mathcal{D}$ on the spaces of effort and flow variables, $\mathcal{E}$ and $\mathcal{F}$ (including the boundary effort and flows $\left.\left(e_{\partial}, f_{\partial}\right)\right)$, which satisfies the relation $\mathcal{D}=\mathcal{D}^{\perp}$. where $\mathcal{D}^{\perp}$ defines the orthogonal subspace of $\mathcal{D}$ with respect to the following pairing:

$$
<<\left(e_{1}, f_{1}\right),\left(e_{2}, f_{2}\right)>>:=<e_{1}\left|f_{2}>+<e_{2}\right| f_{1}>
$$

with $\left(e_{1}, f_{1}\right),\left(e_{2}, f_{2}\right) \in \mathcal{E} \times \mathcal{F}$.

In presence of dissipation the port hamiltonian model can be extended with a specific dissipative port variables $\left(f_{d}, e_{d}\right)$ connected to the system by a map, noted $\mathcal{G}$, as follows

$$
\left(\begin{array}{c}
f \\
e_{d}
\end{array}\right)=\underbrace{\left(\begin{array}{cc}
\mathcal{J} & \mathcal{G} \\
-\mathcal{G}^{\star} & 0
\end{array}\right)}_{\mathcal{J}_{e}}\left(\begin{array}{c}
e \\
f_{d}
\end{array}\right)+\left(\begin{array}{c}
\mathcal{B}\left(f_{\mathcal{Z}}^{c}\right) \\
0
\end{array}\right)
$$

Where $\mathcal{G}^{\star}$ represents the formal adjoint operator of $\mathcal{G}=$ $G_{1} \frac{\partial}{\partial z}$ with $G_{1} \in \mathbb{R}^{2 \times m}$ a constant matrix. The constitutive equation of dissipation is given by $f_{d}=-D e_{d}$ with $D \in \mathbb{R}^{m \times m}$. The extended skew symmetric operator $\mathcal{J}_{e}$ can be written as $\widetilde{P}_{0}+\widetilde{P}_{1} \partial_{z}$ with constant matrices $\widetilde{P}_{1}=\left(\begin{array}{cc}P_{1} & G_{1} \\ G_{1}^{T} & 0\end{array}\right)$ and $\widetilde{P}_{0}=\left(\begin{array}{cc}P_{0} & 0 \\ 0 & 0\end{array}\right)$. We obtain $f=(\mathcal{J}-$ $\left.\mathcal{G} D \mathcal{G}^{\star}\right) e$

A set of corresponding boundary variables $\left(f_{\partial}, e_{\partial}\right)$ can be chosen using the parametrization proposed in Le Gorrec et al. (2005). The power product is then defined with respect to the extended flow and effort variables $e_{e}=$ $\left(e, e_{d}\right)$ and $f_{e}=\left(f, f_{d}\right)$ as follows

$$
\begin{aligned}
<\left[\begin{array}{c}
e_{e} \\
e_{\mathcal{Z}}^{c} \\
e_{\partial}
\end{array}\right] \mid\left[\begin{array}{l}
f_{e} \\
f_{\mathcal{Z}}^{c} \\
f_{\partial}
\end{array}\right]>: & =<e_{e}, f_{e}>_{L^{2}}+<e_{\mathcal{Z}}^{c}, f_{\mathcal{Z}}^{c}>_{L^{2}} \\
& +<e_{\partial}^{b}, f_{\partial}^{b}>_{R^{3}}-<e_{\partial}^{a}, f_{\partial}^{a}>_{R^{3}}
\end{aligned}
$$

Thus the new extended structure $J_{e}$ define a Stokes Dirac structure on the extended effort and flow variables.

\section{IRREVERSIBLE THERMODYNAMICS FOR DISTRIBUTED SYSTEMS}

In the framework of equilibrium thermodynamics under the assumption that there is no mechanical energy the total energy is the internal energy $U$. We consider here the case where the convection takes place. In the case of a homogeneous system formed of $n_{c}$ species in a volume $V$, $U$ is a function of the entropy $S, V$ and the mass $m_{i}$ of species $i$. This internal energy $U$ fulfils the Gibbs relation

$$
\mathrm{d} U=T \mathrm{~d} S-P \mathrm{~d} V+\sum_{i=1}^{n_{c}} \mu_{i} \mathrm{~d} m_{i}
$$

where $T=\partial_{S} U$ is the temperature, $P=-\partial_{V} U$ the pressure and $\mu_{i}=\partial_{m_{i}} U$ the chemical potential of species $i$. From this equation all the thermodynamic properties of the system can be deduced.

As soon as non-equilibrium thermodynamics is considered the Gibbs equation (8) remains valid by again using the local equilibrium assumption. This assumption states that the present state of the homogeneous system in any evolution can be characterized by the same variables as at equilibrium and is independent on the rate of evolution.

As far as distributed parameter systems are concerned, the systems are generally not homogeneous. So a local version of (8) is derived. It is assumed that the system may be decomposed in subsystems small enough to be homogeneous at the macroscopic scale, and large enough, on the microscopic scale, to have many particles in such a way that the macroscopic extensive quantity $A$ keep a well-defined meaning given by:

$$
A(t)=\int_{V} \rho(z, t) a(z, t) \mathrm{d} z
$$

where $\rho$ is the total mass density, $a(z, t)$ the quantity per unit mass of $A(t)$ and $z$ the coordinate space respectively.

Then the local equilibrium hypothesis states that (8) is locally valid with quantities per unit mass as soon as the mass under consideration remains invariant and moves with the mass average velocity $v$ (see Bird (2002)). The Gibbs relation can be then written as:

$$
\mathrm{d} u=T \mathrm{~d} s-P \mathrm{~d}\left(\frac{1}{\rho}\right)+\sum_{i=1}^{n_{c}} \mu_{i} \mathrm{~d} \omega_{i}
$$

The local equilibrium hypothesis for distributed system is expressed in the local form by:

$$
\rho \frac{D u}{D t}=\rho\left(T \frac{D s}{D t}-P \frac{D\left(\frac{1}{\rho}\right)}{D t}+\sum_{i=1}^{n_{c}} \mu_{i} \frac{D \omega_{i}}{D t}\right)
$$

where $\frac{D a}{D t}$ stands for the material derivative $\frac{\partial a}{\partial t}+v \frac{\partial a}{\partial z}$ of the quantity $a$.

Let us now give the integral formulation of equation (11). For simplicity in the remainder of the section we consider that volumes are cylinders of section $\bar{S}$ and that homogeneity properties are assumed such that only the longitudinal coordinate $z$ is necessary. We consider an incompressible fluid phase so the total density $\rho$ is constant.

Then for any moving sub domain strictly included in the physical geometric domain corresponding to the reactor, we obtain the integral form of (11):

$$
\frac{\mathrm{d}}{\mathrm{d} t} \int_{z_{a}+v t}^{z_{b}+v t} \rho u(z, t) \bar{S} \mathrm{~d} z=\int_{z_{a}+v t}^{z_{b}+v t} \frac{D \rho u(z, t)}{D t} \bar{S} \mathrm{~d} z
$$

So we finally obtain the integral form of equation (11): 


$$
\begin{aligned}
\frac{\mathrm{d}}{\mathrm{d} t} \int_{z_{a}+v t}^{z_{b}+v t} \rho u(z, t) \bar{S} \mathrm{~d} z & =\int_{z_{a}+v t}^{z_{b}+v t} T \frac{D \rho s(z, t)}{D t} \bar{S} \mathrm{~d} z \\
& +\sum_{i=1}^{n_{c}} \int_{z_{a}+v t}^{z_{b}+v t} \mu_{i} \frac{D \rho \omega_{i}(z, t)}{D t} \bar{S} \mathrm{~d} z
\end{aligned}
$$

Equation (13) expresses the energy conservation of distributed systems.

So we can consider as energy distributed conjugate variables the following pairings: $(\rho s, T)$ and $\left(\rho \omega_{i}, \mu_{i}\right)$. These pairings are naturally power conjugate with the product rule defined by (13).

\section{PORT HAMILTONIAN FORMULATION OF A TUBULAR REACTOR SYSTEM}

\subsection{Basic dynamical model}

The reactor is assumed to have a length $L$. The axial coordinate is $z$. We suppose isobaric operating conditions. The chemical reaction $r$ involves two species say $A$ and $B: \nu_{A} A \longrightarrow \nu_{B} B$, and $\nu_{A}, \nu_{B}>0$ are the stoichiometric coefficients of the reaction. The reaction kinetics is modeled thanks to the Arrhenius law. It is not necessary to give the kinetics of the reaction. We only assume that this latter depends non linearly on the concentration of species $A$ so it can be expressed also with respect to the chemical potential $\mu_{A}$. We assume that there is no convection and that some diffusion is occurring in the tubular reactor. The diffusion flux $f_{d}^{i}$ (for $i=A, B$ ) is related to the thermodynamic driving force $\partial_{z} \mu_{i}$ as

$$
f_{d}^{i}=-D \partial_{z} \mu_{i}
$$

(see Bird (2002)).

The material balance of the species $\mathrm{i}(\mathrm{i}=A$ or $B$ ) is given by:

$$
\partial_{t} w_{i}=-\partial_{z} f_{d}^{i}-\frac{M_{i} \nu_{i} r}{\rho}
$$

where $M_{i}$ is the molar mass of species $i$.

The energy balance with incompressible and isobaric hypotheses can be expressed with the enthalpy balance:

$$
\partial_{t} h=-\partial_{z} f_{d}^{h}-\frac{q}{\rho}
$$

where $h=u+P \frac{1}{\rho}$ the enthalpy per mass unit. The enthalpy flux due to diffusion is given by

$$
f_{d}^{h}=\sum_{i=A, B} f_{d}^{i} h_{i}
$$

where $h_{i}$ is the specific enthalpy of species $i$ and $q$ is the distributed thermal exchange with the jacket of the reactor. We do not explicit its constitutive law.

The balance of entropy is given by

$$
\partial_{t} s=-\partial_{z} f_{d}^{s}-\frac{q}{\rho T_{j}}+\frac{\sigma_{s}}{\rho}
$$

with $T_{j}$ is the distributed temperature of the jacket, $f_{d}^{s}=\sum_{i=A, B} f_{d}^{i} s_{i}$ is the entropy flux due to diffusion and $s_{i}$ is the specific entropy of species $i$. The irreversible production of entropy is given by:

$$
\begin{array}{r}
\sigma_{s=} \overbrace{q\left(\frac{1}{T_{j}}-\frac{1}{T}\right)}^{\sigma_{e x t}} \overbrace{+\frac{r}{T}\left(\mu_{A} M_{A} \nu_{A}-\mu_{B} M_{B} \nu_{B}\right)}^{\sigma_{r}} \\
\overbrace{-\frac{1}{T} \sum f_{d}^{i} \partial_{z} \mu_{i}-f_{d}^{s} \frac{\partial_{z} T}{T}}^{\sigma_{d}^{\text {mat }}}
\end{array}
$$

Each term of (18) is assumed to be positive in the context of the irreversible thermodynamics Callen (1985); Glansdorff and Prigogine (1971). The first term in (18) corresponds to the exchange with the jacket of the reactor. The second term is due to the chemical reaction. The third and the fourth terms are due to diffusion in the material domain and in the thermal domain respectively. Let us denote $\sigma_{d}=\sigma_{d}^{\text {mat }}+\sigma_{d}^{\text {therm }}$.

\subsection{Port hamiltonian formulation in the Energy vision}

We consider the internal energy $\rho u$ as Hamiltonian function and derive the dynamic model through the material and the entropy balances. These balances are related using the Gibbs equation (8). In the sequel we will show that a Stokes Dirac structure can be defined for diffusion phenomena. In presence of the chemical reaction, this is no more the case due to the nonlinearities depending on the state and the effort variables.

In order to define the Stokes Dirac structure, we introduce the pairing of effort and flow variables as shown in table 1.

Table 1. variables settings

\begin{tabular}{llll} 
flows & \multicolumn{3}{c}{ efforts } \\
\hline$F_{A}$ & $=\partial_{t} \omega_{A}$ & $e_{A}$ & $=\mu_{A}$ \\
$F_{B}$ & $=\partial_{t} \omega_{B}$ & $e_{B}$ & $=\mu_{B}$ \\
$F_{s}$ & $=\partial_{t} s$ & $e_{s}$ & $=T$ \\
$f_{A}$ & $=f_{d}^{A}$ & $E_{A}$ & $=-\partial_{z} \mu_{A}$ \\
$f_{B}$ & $=f_{d}^{B}$ & $E_{B}$ & $=-\partial_{z} \mu_{B}$ \\
$f_{s}$ & $=f_{d}^{s}$ & $E_{s}$ & $=-\partial_{z} T$ \\
$f_{\sigma}$ & $=T$ & $e_{\sigma}$ & $=-\frac{1}{T} \sigma_{d}$ \\
$f_{\mathcal{Z}}^{c}$ & $=\frac{q}{T \rho}$ & $e_{\mathcal{Z}}^{c}$ & $=-T$
\end{tabular}

Let us now consider the vectors $\mathrm{E}$ and $\mathrm{F}$ :

$$
\begin{aligned}
& E=\left(\begin{array}{lllllll}
e_{A} & e_{B} & e_{s} & f_{A} & f_{B} & f_{s} & e_{\sigma}
\end{array}\right)^{T} \\
& F=\left(\begin{array}{lllllll}
F_{A} & F_{B} & F_{s} & E_{A} & E_{B} & E_{s} & f_{\sigma}
\end{array}\right)^{T}
\end{aligned}
$$

with $E \in \mathbf{E}=\left(H^{2}[0, L]\right)^{3} \times\left(H^{1}[0, L]\right)^{3} \times\left(L^{2}[0, L]\right)$ and $F \in \mathbf{F}=\left(L^{2}[0, L]\right)^{3} \times\left(H^{1}[0, L]\right)^{3} \times\left(L^{2}[0, L]\right)$

According to balances $(15,17)$, we have for the autonomous part ( $q$ is considered as an input):

$$
F=J E
$$

with

$$
J=J_{1}+P_{0}(e)
$$

where $J_{1}=P_{1} \partial_{z}$ with

$$
P_{1}=\left(\begin{array}{ccc}
0_{3} & -I_{3} & 0 \\
-I_{3} & 0_{3} & 0 \\
0 & 0 & 0_{1}
\end{array}\right)
$$


where $0_{3}, I_{3} \in \mathbb{R}^{3 \times 3}, 0_{1} \in \mathbb{R}$ represent the zero matrix, the identity matrix and the scalar 0 respectively.

$$
P_{0}(e)=\left(\begin{array}{ccc}
\mathcal{J} & 0_{3} & P \\
0_{3} & 0_{3} & 0 \\
-P^{T} & 0 & 0_{1}
\end{array}\right)
$$

with

$$
\mathcal{J}=\left(\begin{array}{ccc}
0 & 0 & -\gamma_{1} \\
0 & 0 & \gamma_{2} \\
\gamma_{1} & -\gamma_{2} & 0
\end{array}\right)
$$

and $\gamma 1=\frac{\nu_{A} M_{A} r}{T \rho}, \gamma_{2}=\frac{\nu_{B} M_{B} r}{T \rho}$ and $P^{T}=\left(\begin{array}{lll}0 & 0 & -1\end{array}\right)$.

Let us consider the following boundary port variables:

$$
e_{\partial}=\left.\left(\begin{array}{c}
e_{A} \\
e_{B} \\
e_{s}
\end{array}\right)\right|_{0, L}, f_{\partial}=\left.\left(\begin{array}{c}
f_{A} \\
f_{B} \\
f_{s}
\end{array}\right)\right|_{0, L}
$$

Set $\mathcal{F}=\left\{\left[\begin{array}{c}F \\ f_{\mathcal{Z}}^{c} \\ f_{\partial}\end{array}\right] \in \mathbf{F} \times L^{2}[0, L] \times \mathbb{R}^{3 \times\{0, L\}}\right\}$ and $\mathcal{E}=\left\{\left[\begin{array}{c}E \\ e_{\mathcal{Z}}^{c} \\ e_{\partial}\end{array}\right] \in \mathbf{E} \times L^{2}[0, L] \times \mathbb{R}^{3 \times\{0, L\}}\right\}$. Let us endow the subspaces $\mathcal{E}$ and $\mathcal{F}$ with the pairing

$$
\begin{gathered}
<\left[\begin{array}{c}
E \\
e_{\mathcal{Z}}^{c} \\
e_{\partial}
\end{array}\right] \mid\left[\begin{array}{c}
F \\
f_{\mathcal{Z}}^{c} \\
f_{\partial}
\end{array}\right]>:=<E, F>_{L^{2}}+<e_{\mathcal{Z}}^{c}, f_{\mathcal{Z}}^{c}>_{L^{2}} \\
+<e_{\partial}^{L}, f_{\partial}^{L}>_{R^{3}}-<e_{\partial}^{0}, f_{\partial}^{0}>_{R^{3}}
\end{gathered}
$$

Let us note that with the above notations we have:

$$
\sigma_{d}(e)=-\frac{1}{E(3)} E^{T}\left(\begin{array}{ccc}
0_{3} & 0_{3} & 0 \\
0_{3} & I_{3} & 0 \\
0 & 0 & 0_{1}
\end{array}\right) F
$$

Proposition 1. The linear subset $\mathcal{D} \subset \mathcal{E} \times \mathcal{F}$ defined by: $\mathcal{D}=\left\{\left(\left[\begin{array}{c}F \\ f_{\mathcal{Z}}^{c} \\ f_{\partial}\end{array}\right],\left[\begin{array}{c}E \\ e_{\mathcal{Z}}^{c} \\ e_{\partial}\end{array}\right]\right) \in \mathcal{E} \times \mathcal{F} \mid F=J E+\right.$ $\left(\begin{array}{c}\mathcal{B}\left(e_{\mathcal{Z}}^{c}\right) \\ 0 \\ 0\end{array}\right)$ with the distributed input operator $\mathcal{B}=\left(\begin{array}{l}0 \\ 0 \\ 1\end{array}\right)$, $e_{\mathcal{Z}}^{c}=-\left[\begin{array}{lll}\mathcal{B}^{\star} & 0 & 0\end{array}\right] E,\left[\begin{array}{l}f_{\partial} \\ e_{\partial}\end{array}\right](0, L)$ as defined in $(25)$ and with relations (14) and (27) $\}$ is a conservative structure with respect to the symmetry pairing (5). If the reaction terms $\mathcal{J}$ is identically equal to 0 , the subset is a Stokes Dirac structure.

Proof. We can write (20) as a new extension of the operator given in (6) as follows:

$$
\left(\begin{array}{ccc}
\mathcal{J} & \mathcal{G} & P \\
-\mathcal{G}^{\star} & 0_{3} & 0 \\
-P^{T} & 0 & 0_{1}
\end{array}\right)
$$

where $\mathcal{G}=I_{3} \partial_{z}, f_{\mathcal{Z}}^{c}=\frac{q(z, t)}{T(z, t)}$ and its dual effort variable $e_{\mathcal{Z}}^{c}=T(z, t)$.

We split the vector $E$ as follows: $E_{1}=\left(e_{A} e_{B} e_{s}\right)^{T}$, $E_{2}=\left(f_{A} f_{B} f_{s}\right)^{T}, E_{3}=e_{\sigma}$. First we calculate the term $<E, J E>_{L^{2}}$ :

$$
\begin{aligned}
<E, J E>_{L^{2}}= & \int_{0}^{L}\left(E_{1}^{T} \mathcal{J} E_{1}+E_{1}^{T} \mathcal{G} E_{2}-E_{2}^{T} \mathcal{G}^{\star} E_{1}\right. \\
& \left.+E_{1}^{T} P E_{3}-E_{3}^{T} P^{T} E_{1}\right) d z
\end{aligned}
$$

Due to the skew symmetry of $\mathcal{J}$ we have $E_{1}^{T} \mathcal{J} E_{1}=0$ and the scalar term $E_{1}^{T} P E_{3}$ is equal to $E_{3}^{T} P^{T} E_{1}$. We obtain

$$
\begin{aligned}
<E, J E>_{L^{2}}= & \int_{0}^{L}\left(E_{1}^{T} \mathcal{G} E_{2}-E_{2}^{T} \mathcal{G}^{\star} E_{1}\right) d z \\
= & \int_{0}^{L}-\left(e_{A}\left(\partial_{z} f_{A}\right)+e_{B}\left(\partial_{z} f_{B}\right)+e_{s}\left(\partial_{z} f_{s}\right) d z\right. \\
& -f_{A}\left(\partial_{z} e_{A}\right)+f_{B}\left(\partial_{z} e_{B}\right)+f_{s}\left(\partial_{z} e_{s}\right) d z \\
= & \int_{0}^{L}\left(-\partial_{z}\left(e_{A} f_{A}+e_{B} f_{B}+e_{s} f_{s}\right)\right) d z \\
= & -\left.e_{\partial}^{T} f_{\partial}\right|_{L}+\left.e_{\partial}^{T} f_{\partial}\right|_{0} \\
= & -<e_{\partial, L}, f_{\partial, L}>_{R^{3}}+<e_{\partial, 0}, f_{\partial, 0}>_{R^{3}}(30)
\end{aligned}
$$

As a result,

$$
<E, J E>_{L^{2}}+<e_{\partial}, f_{\partial}>_{R^{3}}=0
$$

Thus the total power product including the distributed external power flow can be written

$$
<E, F>_{L^{2}}+<e_{\mathcal{Z}}^{c}, f_{\mathcal{Z}}^{c}>_{L^{2}}+<e_{\partial}, f_{\partial}>_{R^{3}}=0
$$

This result shows that the energy of the system is conserved in accordance to the first thermodynamic law.

Remark:Let us now focus on the reaction part only. The reaction part can be written as an irreversible port hamiltonian system as suggested in Ramirez et al. (2012). The obtained model is called quasi-Hamiltonian system due to the dependency of the structure matrix $\mathcal{J}$ on the effort variables $\partial_{x} u(x)$ and the consequence is that the dirac structure is destroyed. In a similar way the chemical reaction can be written as:

$$
\partial_{t} x=R\left(x, \partial_{x} u, \partial_{x} s\right) J_{r} \partial_{x} u(x)
$$

where $x^{T}=\left(\begin{array}{lll}\omega_{A} & \omega_{B} & s\end{array}\right)$

where $J_{r}$ a constant skew-symmetric matrix given by:

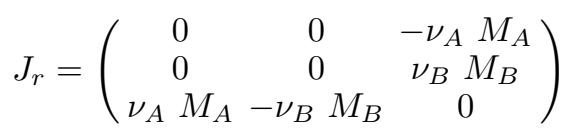

and $\mathrm{R}$ is composed of a positive definite function and a poisson bracket

$$
R\left(x, \frac{\partial u}{\partial x}, \frac{\partial s}{\partial x}\right)=\gamma\left(x, \frac{\partial u}{\partial x}\right)\{s, u\}_{J_{r}}
$$

with

$$
\{s, u\}_{J_{r}}=\left(\partial_{x} s\right)^{T} J_{r}\left(\partial_{x} u\right)=\left(\begin{array}{lll}
0 & 0 & 1
\end{array}\right) J_{r}\left(\begin{array}{c}
\mu_{A} \\
\mu_{B} \\
T
\end{array}\right)=\mathcal{A}
$$

where $\mathcal{A}=\nu_{1} M_{1} \mu_{A}-\nu_{2} M_{2} \mu_{B}$ is the chemical affinity of the reaction which defines the chemical driving force.

The function $\gamma$ is given by

$$
\gamma\left(x, \frac{\partial U}{\partial x}\right)=\frac{r(T)}{T \rho}
$$




\subsection{Port hamiltonian formulation in the Entropy vision}

The formulation of entropy vision is based on the Gibbs equation in entropy vision with $h=u+P \frac{1}{\rho}$. We have $d s=\frac{1}{T} d h-\sum \frac{\mu_{i}}{T} \omega_{i}$. We formulate the system as a Port Hamiltonian system with $-s$ as the Hamiltonian. The state variables of the dynamic part are the mass fraction of species and the enthalpy. So in this case the effort variables are $-\frac{1}{T}$ and $\frac{\mu_{i}}{T}$.

Firstly let us recall how the chemical reaction is treated (see Hoang et al. (2011) for details):

Let us note that the flow term of the reaction can be written as

$$
\left(\begin{array}{c}
-\frac{\nu_{A} M_{A} r}{\rho} \\
\frac{\nu_{B} M_{B} r}{\rho}
\end{array}\right)=\frac{r T}{2 \rho}\left(J_{r}+R_{r}\right)\left(\begin{array}{c}
\frac{\mu_{A}}{T} \\
\frac{\mu_{B}}{T}
\end{array}\right)
$$

$J_{r}=\left(\begin{array}{cc}0 & -\alpha \\ \alpha & 0\end{array}\right)$ and $R_{r}=\left(\begin{array}{cc}\frac{M_{A} \nu_{A}}{\mu_{A}} & \beta \\ \beta & -\frac{M_{B} \nu_{B}}{\mu_{B}}\end{array}\right)$ where $\alpha=\frac{M_{A} \nu_{A}}{2 \mu_{B}}+\frac{M_{B} \nu_{B}}{2 \mu_{A}}$ and $\beta=\frac{M_{A} \nu_{A}}{2 \mu_{B}}-\frac{M_{B} \nu_{B}}{2 \mu_{A}}$.

Let us consider the effort and flow variables of Table 2:

Table 2. variables settings

\begin{tabular}{ll} 
Flows & efforts \\
\hline$F_{A}=\partial_{t} \omega_{A}$ & $e_{A}=\frac{\mu_{A}}{T}$ \\
$F_{B}=\partial_{t} \omega_{B}$ & $e_{B}=\frac{\mu_{B}}{T}$ \\
$F_{h}=\partial_{t} h$ & $e_{h}=-\frac{1}{T}$ \\
$f_{A}=f_{d}^{A}$ & $E_{A}=-\frac{1}{2} \partial_{z}\left(\frac{\mu_{A}}{T}\right)$ \\
$f_{B}=f_{d}^{B}$ & $E_{B}=-\frac{1}{2} \partial_{z}\left(\frac{\mu_{B}}{T}\right)$ \\
$f_{h}=f_{d}^{h}$ & $E_{h}=-\frac{1}{2} \partial_{z}\left(\frac{-1}{T}\right)$ \\
$f_{\sigma}^{A}=-\frac{1}{2} f_{d}^{A}$ & $e_{\sigma}^{A}=-\partial_{z}\left(\frac{\mu_{A}}{T}\right)$ \\
$f_{\sigma}^{B}=-\frac{1}{2} f_{d}^{B}$ & $e_{\sigma}^{B}=-\partial_{z}\left(\frac{\mu_{B}}{T}\right)$ \\
$f_{\sigma}^{h}=-\frac{1}{2} f_{d}^{h}$ & $e_{\sigma}^{h}=-\partial_{z}\left(\frac{-1}{T}\right)$ \\
$f_{\mathcal{Z}}^{c}=\frac{q}{\rho}$ & $e_{\mathcal{Z}}^{c}=-\frac{1}{T}$ \\
\hline
\end{tabular}

and consider the vector $E$ and $F$ :

$$
\begin{aligned}
& E=\left(\begin{array}{lllllllll}
e_{A} & e_{B} & e_{h} & f_{d}^{A} & f_{d}^{B} & f_{d}^{h} & e_{\sigma}^{A} & e_{\sigma}^{B} & e_{\sigma}^{h}
\end{array}\right)^{T} \\
& F=\left(\begin{array}{lllllllll}
F_{A} & F_{B} & F_{h} & E_{A} & E_{B} & E_{h} & f_{\sigma}^{A} & f_{\sigma}^{B} & f_{\sigma}^{h}
\end{array}\right)^{T}
\end{aligned}
$$

With $E \in \mathbf{E}=\left(H^{2}[0, L]\right)^{3} \times\left(H^{1}[0, L]\right)^{3} \times\left(L^{2}[0, L]^{3}\right)$ and $F \in \mathbf{F}=\left(L^{2}[0, L]\right)^{3} \times\left(H^{1}[0, L]\right)^{3} \times\left(L^{2}[0, L]\right)^{3}$

From the mass balance (15) and the entropy balance (16) the system can be written in the following compact form:

$$
F=(J-R) E+\mathcal{B}_{e}\left(f_{\mathcal{Z}}^{c}\right)
$$

where

$$
\begin{gathered}
J=J_{1}+P_{0}(e), \quad J_{1}=\underbrace{\left(\begin{array}{ccc}
0_{3} & -I_{3} & 0_{3} \\
-I_{3} & 0_{3} & 0_{3} \\
0_{3} & 0_{3} & 0_{3}
\end{array}\right)}_{P_{1}} \partial z \\
P_{0}(e)=\left(\begin{array}{lll}
\mathcal{J} & 0_{3} & 0_{3} \\
0_{3} & 0_{3} & 0_{3} \\
0_{3} & 0_{3} & 0_{3}
\end{array}\right) \text { with } \mathcal{J}=\alpha \frac{r T}{2 \rho}\left(\begin{array}{ccc}
0 & -1 & 0 \\
1 & 0 & 0 \\
0 & 0 & 0
\end{array}\right)
\end{gathered}
$$

$$
R=\left(\begin{array}{ccc}
\mathcal{R} & 0_{3} & 0_{3} \\
0_{3} & 0_{3} & \frac{1}{2} I_{3} \\
0_{3} & \frac{1}{2} I_{3} & 0_{3}
\end{array}\right), \quad \mathcal{R}=\left(\begin{array}{ccc}
\frac{r T}{2 \rho} \frac{M_{A} \nu_{A}}{\mu_{A}} & \frac{r T}{2 \rho} \beta & 0 \\
\frac{r T}{2 \rho} \beta & -\frac{r T}{2 \rho} \frac{M_{B} \nu_{B}}{\mu_{B}} & 0 \\
0 & 0 & 0
\end{array}\right)
$$

and the extended input operator

$$
\mathcal{B}_{e}\left(f_{\mathcal{Z}}^{c}\right)=\left(\begin{array}{c}
\mathcal{B}\left(f_{\mathcal{Z}}^{c}\right) \\
0 \\
0
\end{array}\right)
$$

with $\mathcal{B}=\left(\begin{array}{l}0 \\ 0 \\ 1\end{array}\right)$ and $f_{\mathcal{Z}}^{c}=\frac{q(z, t)}{\rho}, e_{\mathcal{Z}}^{c}=\frac{-1}{T(z, t)}$. Let us consider the following boundary port variables:

$$
e_{\partial}=\left.\left(\begin{array}{c}
e_{A} \\
e_{B} \\
e_{h}
\end{array}\right)\right|_{0, L}, f_{\partial}=\left.\left(\begin{array}{c}
f_{A} \\
f_{B} \\
f_{h}
\end{array}\right)\right|_{0, L}
$$

Set $\mathcal{F}=\left\{\left[\begin{array}{c}F \\ f_{\mathcal{Z}}^{c} \\ f_{\partial}\end{array}\right] \in \mathbf{F} \times L^{2}[0, L] \times \mathbb{R}^{3 \times\{0, L\}}\right\}$ and

$\mathcal{E}=\left\{\left[\begin{array}{c}E \\ e_{\mathcal{Z}}^{c} \\ e_{\partial}\end{array}\right] \in \mathbf{E} \times L^{2}[0, L] \times \mathbb{R}^{3 \times\{0, L\}}\right\}$. Let us endow the subspaces $\mathcal{E}$ and $\mathcal{F}$ with the pairing analog to the one given in (26).

Proposition 2. The extended system defined in (39-43), where the term $P_{0}(e)$ is neglected, defines a dissipative port Hamiltonian system with $-s$ as Hamiltonian function. The associated Stokes-Dirac structure is given by the skew symmetric operator $J_{1}$. The dissipation term due to irreversibility is equal to $-\sigma_{r}-\sigma_{d}^{\text {mat }}-\sigma_{d}^{\text {therm }}$. Now, by considering the term $P_{0}(e)$ the extended system (39-43) defines a quasi-dissipative Hamiltonian system with the same dissipation term.

Proof. It is obvious that without $P(e)$, the operator $\mathcal{J}_{1}$ defines a Stokes Dirac structure. Hereafter we check that the proposed structure is power conservative. We can write the structure $J$ defined in (40) as a new extension of the operator given in (6) as follows:

$$
\left(\begin{array}{ccc}
\mathcal{J} & \mathcal{G} & 0 \\
-\mathcal{G}^{\star} & 0 & 0 \\
0 & 0 & 0
\end{array}\right)
$$

where $\mathcal{J}=\alpha \frac{r T}{2 \rho}\left(\begin{array}{ccc}0 & -1 & 0 \\ 1 & 0 & 0 \\ 0 & 0 & 0\end{array}\right), \mathcal{G}=-I_{3} \partial_{z}$.

Let us compute $\left\langle E, J E>_{L^{2}}\right.$. For this ,we split $E$ as $E_{1}=\left(e_{A} e_{B} e_{h}\right)^{T}, E_{2}=\left(f_{A} f_{B} f_{h}\right)^{T}, E_{3}=\left(e_{\sigma}^{A} e_{\sigma}^{B} e_{\sigma}^{h}\right)^{T}$. First we calculate the term $<E, J E>_{L^{2}}$ :

$$
<E, J E>_{L^{2}}=\int_{0}^{L}\left(E_{1}^{T} \mathcal{J} E_{1}+E_{1}^{T} \mathcal{G} E_{2}-E_{2}^{T} \mathcal{G}^{\star} E_{1}\right) d z
$$

Due to the skew symmetry of $\mathcal{J}$ we have $E_{1}^{T} \mathcal{J} E_{1}=0$. We finally obtain: 


$$
\begin{aligned}
<E, J E>_{L^{2}}= & \int_{0}^{L} E_{1}^{T} \mathcal{G} E_{2}-E_{2}^{T} \mathcal{G}^{\star} E_{1} \\
= & \int_{0}^{L}-\left(e_{A}\left(\partial_{z} f_{A}\right)+e_{B}\left(\partial_{z} f_{B}\right)+e_{h}\left(\partial_{z} f_{h}\right) d z\right. \\
& -f_{A}\left(\partial_{z} e_{A}\right)+f_{B}\left(\partial_{z} e_{B}\right)+f_{h}\left(\partial_{z} e_{h}\right) d z \\
= & \int_{0}^{L}\left(-\partial_{z}\left(e_{A} f_{A}+e_{B} f_{B}+e_{h} f_{h}\right) d z\right. \\
= & -\left.e_{\partial}^{T} f_{\partial}\right|_{L}-\left|+e_{\partial}^{T} f_{\partial}\right|_{0} \\
= & -<e_{\partial, L}, f_{\partial, L}>_{R^{3}}+<e_{\partial, 0}, f_{\partial, 0}>_{R^{3}}(45)
\end{aligned}
$$

Thus the total power product including the distributed external power flow can be written

$$
<E, F>_{L^{2}}+<e_{\mathcal{Z}}^{c}, f_{\mathcal{Z}}^{c}>_{L^{2}}+<e_{\partial}, f_{\partial}>_{R^{3}}=0
$$

This shows that the structure $J$ of the system 39 , the chemical reaction part $\mathcal{J}$, defines a Stokes Dirac structure. It represents the conservative part of the system. Let us show that the nonlinear symmetric matrix $R$ gives rise to dissipation. For this purpose we show that $\langle E, R E\rangle_{L^{2}}=$ $\sigma_{d}+\sigma_{r}$. Let us note that in this context the irreversible entropy production $\sigma_{d}^{\text {therm }}$ can be expressed in function of the enthalpy flux $f_{d}^{h}=T f_{d}^{s}+\mu_{A} f_{d}^{A}+\mu_{B} f_{d}^{B}$. We obtain

$$
\begin{aligned}
& \sigma_{d}^{\text {therm }}=-f_{d}^{h}\left(\frac{1}{T^{2}} \partial_{z} T\right)+f_{d}^{A}\left(\frac{\mu_{A}}{T^{2}} \partial_{z} T\right)+f_{d}^{B}\left(\frac{\mu_{B}}{T^{2}} \partial_{z} T\right) \\
& <E, R E>_{L^{2}}=\int_{0}^{L}\left(E^{T} R E\right) d z \\
& =\int_{0}^{L} e_{A} \frac{r T}{2 \rho} \frac{M_{A} \nu_{A}}{\mu_{A}} e_{A}+e_{A} \frac{r T}{2 \rho} \beta e_{B} \\
& \underbrace{e_{B} \frac{r T}{2 \rho} \beta e_{A}+e_{B}-\frac{r T}{2 \rho} \frac{M_{B} \nu_{B}}{\mu_{B}} e_{B}}_{R_{1}} \\
& \underbrace{-f_{d}^{A} \partial_{z}\left(\frac{\mu_{A}}{T}\right)-f_{d}^{B} \partial_{z}\left(\frac{\mu_{B}}{T}\right)+f_{d}^{h} \partial_{z}\left(\frac{1}{T}\right)}_{R_{2}} d z
\end{aligned}
$$

Finally we obtain:

- $R_{1}=\frac{r}{\rho T}\left(M_{A} \mu_{A} \nu_{A}-M_{B} \mu_{B} \nu_{B}\right)=\sigma_{r}$.

- Using the equality $\partial_{z}\left(\frac{\mu_{i}}{T}\right)=-\frac{\mu_{i}}{T^{2}} \partial_{z} T+\frac{1}{T} \partial_{z} \mu_{i}$ for $i=A, B$, we finally obtain thanks to (47) and (18)

$$
\begin{aligned}
R_{2}= & f_{d}^{A} \frac{\mu_{A}}{T^{2}} \partial_{z} T-f_{d}^{A} \frac{1}{T} \partial_{z} \mu_{A}+f_{d}^{B} \frac{\mu_{B}}{T^{2}} \partial_{z} T \\
& -f_{d}^{B} \frac{1}{T} \partial_{z} \mu_{B}+f_{d}^{h} \partial_{z}\left(\frac{1}{T}\right)=\sigma_{d}
\end{aligned}
$$

As a result , $\langle E, R E\rangle_{L^{2}}>0$. Thus the system defined in (39-43) defines a quasi-dissipative port Hamiltonian system with $-s$ as Hamiltonian function.

\section{CONCLUSION}

This paper is a first attempt to the port hamiltonian representation of a tubular reactor. It is shown how diffusion transport phenomena and chemical reactions can be represented in the energy based representation as well as in the entropy based representation. It is shown that there are two extensions of the port variables vector. The first one is classical and represents the dissipation. For the energy based representation the second extension correspond to consider a dual representation of the thermal domain: the temperature is considered as a flow. For the entropy based representation the extension is also the dual of the dissipation. It remains to show how the convection phenomenon can be represented. These formulations, specially the entropy one, are well suitable for control purposes using the passivity based methods availability function introduced in Ruszkowski (2005) and used for the control of Continuous stirred tank reactor Hoang et al. (2011).

\section{ACKNOWLEDGEMENTS}

This contribution has been done within the context of the French National Research Agency sponsored project HAMECMOPSYS (ANR-11-BS03-0002)

\section{REFERENCES}

A. Baaiu, F. Couenne, L. Lefevre, Y. Le Gorrec, M. Tayakout Structure-preserving infinite dimensional model reduction: Application to adsorption processes Journal of Process Control, 19:3, 394-404, 2009.

R.B. Bird, W.E. Stewart, and E.N. Lightfoot. Transport phenomena New York: John Wiley and Sons, 2nd edition, 2002.

H.B. Callen. Thermodynamics and an introduction to thermostatics. JohnWiley \& Sons Inc, 2nd ed. New York, 1985.

F. Couenne, C. Jallut, B. Maschke, M. Tayakout and P. C. Breedveld Structured modeling for processes : a thermodynamical network theory. Computers and Chemical Engineering, 32(6) :1120-1134, 2008.

T.J. Courant. Dirac manifolds Trans.American Mathematical society,319:2,631-661,1990.

I. Dorfman. Dirac structures and integrability of nonlinear evolution equations John Wiley, Chichester,1993.

P. Glansdorff and I. Prigogine (1971). Thermodynamic theory of structure, stability and fluctuations. WileyInterscience.

H. Hoang, F. Couenne, C. Jallut and Y. Le Gorrec The port Hamiltonian approach to modeling and control of Continuous Stirred Tank Reactors Journal of Process Control, 21:10, 1449-1458, 2011.

Y. Le Gorrec, H. Zwart, and B. Maschke. Dirac structures and boundary control systems associated with skewsymmetric differential operators. SIAM Journal on Control and Optimization, 42(5):1864-1892, 2005.

B. Maschke and A. J. Van der Schaft, Advanced Topics in Control Systems Theory, chapter: Compositional modelling of distributed-parameter systems, Lecture Notes on Control and Information Sciences. Springer, pp 115154, 2005.

H. Ramirez and B. Maschke and D. Sbarbaro, Irreversible port Hamiltonian systems. accepted to 4th IFAC Workshop. on. Lagrangian and Hamiltonian Methods for Non Linear Control. Bertinoro, Italy 29-31 August 2012.

M. Ruszkowski, V. Garcia-Osorio, and B.E. Ydstie. Passivity based control of transport reaction systems. AIChE Journal, 51:31473166, 2005.

A.J. Van der schaft and B. Maschke Hamiltonian formulation of distributed parameter systems with boundary energy flows Journal of Geometry and physics, 42:1/2,166194,2002 . 